\title{
Should adjuvant radiotherapy be used in patients with early stage Hodgkin's lymphoma? A vote for yes
}

\author{
Ewelina Łata-Woźniak1,2, Aleksandra Łacko1,2

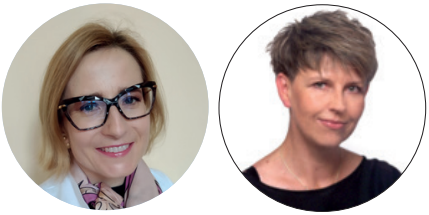 \\ 'Department of Oncology, Wroclaw Medical University, Wroclaw, Poland \\ ${ }^{2}$ Lower Silesian Oncology Center, Wroclaw, Poland
}

Hodgkin's lymphoma ( $\mathrm{HL}$ ) belongs to the most radiosensitive and chemosensitive cancers. Combined modality therapy is the preferred treatment for patients with classical favorable early-stage HL. However, late toxicity still remains an issue. A modern approach in HL radiotherapy includes implementation of sophisticated and dedicated delivery techniques together with the lower doses and smaller fields, which allow for reduction of early and late toxicity. In recent years, the question on the need for complementary radiotherapy in the early stages of Hodgkin's lymphoma has been increasingly raised. The aim of the present review is to discuss the current role of radiotherapy and its potential future developments, with a focus on major clinical trials.

NOWOTWORY J Oncol 2020; 70, 1: 29-32

Key words: Hodgkin's lymphoma, chemotherapy, radiotherapy, combined modality treatment

\section{Introduction}

Hodgkin's lymphoma $(\mathrm{HL})$ belongs to the most radiosensitive and chemosensitive cancers. Most frequently young persons, 20-40 years old, suffer from it [1]. In most patients, however, the disease is diagnosed in early stages, which allows for effective recovery and long-term survival.

The key role in the therapy of early stages Hodgkin's lymphoma is played by radiotherapy. Historically, it was the first method of treatment for this disease. Demonstrating the advantage of combined treatment for many years has established a scheme of standard treatment of this disease. The role of chemotherapy alone at early stages has not been precisely defined and has been the subject of endless discussions for many years.

\section{Review of the main studies on the combined treatment of early stage Hodgkin's lymphoma}

Research by the German Lymphoma Group - German Hodgkin Study Group (GHSG), has established standards of management in stage I and II according to the Ann Arbor classification. On the basis of HD10 trial it was found that in patients with favorable prognostic factors 2 cycles of ABVD chemotherapy (doxorubicin, bleomycin, vinblastine, dacarbazine) with adjuvant involved field radiotherapy (IF-RT) at the dose of 20 Gy are equally effective and less toxic than 3 cycles of ABVD with radiotherapy at the dose of 30 Gy [2]. In turn in patients with adverse prognostic factors, a scheme consisting of 4 ABVD cycles or in younger patients (under 60 years of age) - also chemotherapy according to BEACOPP (bleomycin, etoposi- 
de, doxorubicin, cyclophosphamide, vincristine, procarbazine, prednisone) and IF-RT at a dose of $30 \mathrm{~Gy}$ is the recommended standard of treatment [3].

According to the recommendations of the European Society for Medical Oncology (ESMO), the standard procedure is combined treatment, including chemotherapy with radiotherapy [1]. Modern methods of treatment provide very high percentage of cured patients in this group. Therefore, late treatment complications are an increasing therapeutic problem. Late complications of radiotherapy are widely known, mainly in the context of secondary cancers. In turn, data on the long-term toxicity of systemic treatment are less known. The introduction of modern, highly effective and less toxic chemotherapy schemes makes radiotherapy appear to be an old-fashioned method. Hence the question: can we give up the adjuvant irradiation in early stage Hodgkin's lymphoma patients?

\section{Chemotherapy alone versus combined treatment}

The first reports comparing chemotherapy alone with combined treatment showed better control of the disease in patients treated with radiotherapy [4-6]. According to some, such a difference applies only to patients at an early stage with favorable prognostic factors, according to others it is true only in patients with unfavorable prognosis [4-6]. An unquestionable disadvantage of early research in this field are the outdated methods of treatment. These include the previously used large irradiation fields and the lack of PET diagnostic imaging, both during the initial assessment of the progress and to evaluate the metabolic response after chemotherapy. The data obtained from the above-mentioned studies are quite ambiguous and have opened a debate on the necessity of adjuvant radiotherapy in patients with early Hodgkin's lymphoma, a debate that is still ongoing.

Several recent studies have attempted to make a contribution to this discussion [7-9]. An integral part of the protocol was the PET imaging, which was used to assess the stage of the disease and to evaluate early cancer treatment response. In the experimental arm of those studies were patients with complete remission after 2-3 cycles of systemic treatment, who were randomized to chemotherapy alone or combined treatment. According to the above scheme 3 large, randomized trials were conducted: RAPID (UK NCRI), HD16 (GHSG) and H1OF/U (EORTC/GELA/FIL) [10]. And while the main concept of these trials was similar, they differed in several details.

Firstly, none of them applied the current standard of treatment as the control arm. Secondly, the H10 trial introduced a limited irradiation volume, namely the involved-node radiotherapy (IN-RT). In addition, other studies used a conservative method of radiotherapy, i.e. involved-field radiotherapy (IF-RT). All of them also differ in terms of the doses used and, importantly, only in the GHSG HD16 trial the "modern" radiation therapy doses were used, i.e. 20 Gy in patients with favorable prognostic factors. Differences also apply to chemotherapy. Only in the HD16 trial the patients with favorable prognostic factors received 2 ABVD cycles. In other cases, patients received at least 3 cycles of chemotherapy according to the ABVD scheme.

In the group of patients treated with chemotherapy alone, a significant difference in time free from progression was shown. In case of the RAPID study, after 3 years of observation, the difference was 3.8\% in favor of combined treatment. In the H10 study, the 5-year time free from progression was $99 \%$ and $87.1 \%$, respectively, in the group treated with combined treatment vs. chemotherapy alone. It should be emphasized that the increased risk of relapse did not translate in both studies to worse overall survival in the group of patients treated with chemotherapy alone. No HD16 results have been published so far, but early analyses presented in 2018 in the form of an abstract at the convention of the American Society of Hematology suggest similar results.

In 2017 Cochrane's meta-analysis of combined treatment for patients with early Hodgkin's lymphoma was published [11]. Its main conclusions are comparable to the results of the above-mentioned studies. Namely, when an identical number of courses of chemotherapy was administered in both arms, no difference was observed in overall survival (OS) in patients treated with chemotherapy alone compared to patients treated with combined treatment. In patients treated with chemotherapy alone a shorter progression free survival (PFS) was observed. Significantly, there were no differences in mortality rates associated with infections, secondary cancers and cardiological diseases. As a different number of chemotherapy courses were applied in both arms, it is difficult to draw clear conclusions about PFS and OS due to poor quality of scientific evidence and heterogeneity of studies. In a subgroup of patients with early Hodgkin's lymphoma and with a favorable prognosis, the advantage of combined therapy in the context of PFS was demonstrated. However, in patients with adverse prognostic factors, such an advantage has not been demonstrated.

The above-mentioned studies have not been designed in an optimal way, i.e. in a way that would allow to draw a clear conclusion that chemotherapy alone is equally effective in comparison with current standards of combined treatment in patients with early Hodgkin's lymphoma with a favorable prognosis.

\section{The role of PET}

At this point, it is also important to mention the key role of PET imaging as a tool to assess the response to treatment. The value of metabolic regression assessment after 2-3 courses of chemotherapy in patients with early stage without risk factors is unclear and retrospective analyses provide contradictory results. However, the majority of scientists are of the opinion that such a study should be performed in this group of patients 
[12-16]. In contrast, in patients with more advanced stages (stage II with adverse prognostic factors and stages III and IV according to Ann Arbor), interim PET after 2-4 cycles of chemotherapy is a sensitive prognostic factor $[17,18]$.

It is also worth noting that in large randomized trials, the evaluation of PET scans was verified by a panel of experts. In everyday practice, the standard is to rely on an independent description of a nuclear medicine specialist, which may cause some differences in the interpretation of results. Is this a sufficient parameter to assess the severity of the disease? It turns out that not necessarily, because more and more data published in the literature proves that other parameters, such as metabolic tumor volume (MTV) and total lesion glycolysis (TLG), can also be more objective indicators [19].

\section{Innovations in radiotherapy and strategies to reduce radiation toxicity}

As for the modern approach to radiotherapy, which consists of reducing the size of irradiation fields and reducing doses of ionizing radiation, these have resulted in lower expected toxicity of the treatment. Extended field radiotherapy (EF-RT) techniques have been replaced by the techniques of irradiating the region of originally involved lymph nodes, involved field radiotherapy (IF-RT), which provided comparable results while reducing toxicity $[20,21]$. Currently, in accordance with the recommendations of the International Lymphoma Radiation Oncology Group (ILROG), only the area of the originally involved sites should be irradiated: involved site radiotherapy (ISRT) [22]. To date, there are no prospective randomized studies comparing ISRT with IFRT, although more and more reports suggest that field size reduction does not adversely affect the risk of relapse [22]. The ongoing GHSG HD17 study, for which recruitment closed at the end of 2019, is likely to provide an answer to this question. The current guidelines, both by European Society for Medical Oncology (ESMO) and National Comprehensive Cancer Network (NCCN), recommend using ISRT.

In recent years we have also witnessed an extremely rapid development of radiotherapy. Current techniques allow not only to precisely determine the target volume (fusion of localization tomography with MR or PET scan), but also to precisely locate the irradiated area (image-guided radiotherapy, IGRT). IGRT techniques include imaging obtained by using electronic portal imaging device (EPID) systems, 2D-2D kV, KV-CBCT, MV$-C B C T, M V C T$ or ultrasound examination. One of the modern radiotherapy technique is also $4 \mathrm{D}$ radiotherapy, where the fourth dimension is time. An incredible advantage of this method, especially in the case of lesions located in the chest area, is the adaptation to the change of the target volume position during a treatment session. And what is additionally important, over the last dozen or so years the method of radiotherapy treatment has also changed: a traditional 3D-CRT conformal technique (conformal radiotherapy) is being replaced by IMRT (intensity modulated radiotherapy) techniques.

All these techniques enabled more conformal dose distribution to the target volume and reduction of doses in critical organs, which directly translated into lower toxicity of radiotherapy [23].

\section{Summary}

It should be emphasized that patients diagnosed with relapse require intensive second line treatment, often with autologous hematopoietic cells transplantation. Such treatment may result in significant early and late toxicity, often exceeding that of the primary combined treatment. It is estimated that only half of them will achieve long-term remission of the disease.

In conclusion, due to the lack of convincing proofs to support chemotherapy alone, the standard of treatment of the early stages of Hodgkin's lymphoma is still combined therapy. ESMO guidelines for the early stages of Hodgkin's lymphoma recommend combined therapy. NCCN guidelines, on the other hand, allow for chemotherapy alone only in a narrow group of patients who meet all favorable prognosis criteria. Although modern radiotherapy techniques have the potential to reduce the risk of late complications, longer observations are still necessary, if only to confirm this thesis. And, equally importantly, whenever possible, patients should be allowed to participate in prospective randomized clinical trials.

Conflict of interest: none declared

Ewelina Łata-Woźniak

Wroclaw Medical University

Department of Oncology

Plac Hirszfelda 12

53-413 Wrocław, Poland

e-mail:ewelina.lata-wozniak@umed.wroc.pl

\section{Received and accepted: $5 \mathrm{Jul} 2019$}

We are presenting one of the voices in the debate. The other participant in the debate has not presented their article.

\section{References}

1. Eichenauer DA, Aleman BMP, André M, et al. ESMO Guidelines Committee. Hodgkin lymphoma: ESMO Clinical Practice Guidelines for diagnosis, treatment and follow-up. Ann Oncol. 2018; 29(Suppl 4): iv19-iv29, doi: 10.1093/annonc/mdy080, indexed in Pubmed: 29796651.

2. Engert A, Plütschow A, Eich $\mathrm{HT}$, et al. Reduced treatment intensity in patients with early-stage Hodgkin's lymphoma. N Engl J Med. 2010; 363(7): 640-652, doi: 10.1056/NEJMoa1000067, indexed in Pubmed: 20818855.

3. Eich HT, Diehl V, Görgen H, et al. Intensified chemotherapy and dose-reduced involved-field radiotherapy in patients with early unfavorable Hodgkin's lymphoma: final analysis of the German Hodgkin Study Group HD11 trial. J Clin Oncol. 2010; 28(27): 4199-4206, doi: 10.1200/ JCO.2010.29.8018, indexed in Pubmed: 20713848.

4. Dörffel W, Rühl U, Lüders $\mathrm{H}$, et al. Treatment of children and adolescents with Hodgkin lymphoma without radiotherapy for patients in complete remission after chemotherapy: final results of the multinational trial GPOH-HD95. J Clin Oncol. 2013; 31(12): 1562-1568, doi: 10.1200/ JCO.2012.45.3266, indexed in Pubmed: 23509321. 
5. Wolden SL, Chen Lu, Kelly KM, et al. Long-term results of CCG 5942: a randomized comparison of chemotherapy with and without radiotherapy for children with Hodgkin's lymphoma--a report from the Children's Oncology Group. J Clin Oncol. 2012; 30(26): 3174-3180, doi: 10.1200/JCO.2011.41.1819, indexed in Pubmed: 22649136.

6. Eichenauer DA, Becker I, Monsef I, et al. Secondary malignant neoplasms, progression-free survival and overall survival in patients treated for Hodgkin lymphoma: a systematic review and meta-analysis of randomized clinical trials. Haematologica. 2017; 102(10): 1748-1757, doi: 10.3324/haematol.2017.167478, indexed in Pubmed: 28912173.

7. Raemaekers JMM, André MPE, Federico M, et al. Omitting radiotherapy in early positron emission tomography-negative stage I/II Hodgkin lymphoma is associated with an increased risk of early relapse: Clinical results of the preplanned interim analysis of the randomized EORTC/ LYSA/FIL H10 trial. J Clin Oncol. 2014; 32(12): 1188-1194, doi: 10.1200/ JCO.2013.51.9298, indexed in Pubmed: 24637998.

8. Radford J, Illidge T, Counsell N, et al. Results of a trial of PET-directed therapy for early-stage Hodgkin's lymphoma. N Engl J Med. 2015; 372(17): 1598-1607, doi: 10.1056/NEJMoa1408648, indexed in Pubmed: 25901426.

9. Fuchs M, Goergen H, Kobe C, et al. PET-Guided Treatment of Early-Stage Favorable Hodgkin Lymphoma: Final Results of the International, Randomized Phase 3 Trial HD16 By the German Hodgkin Study Group. Blood. 2018; 132(Supplement 1): 925-925, doi: 10.1182/ blood-2018-99-114519.

10. Svane IM, Pedersen AE, Johansen JS, et al. Vaccination with p53 peptide-pulsed dendritic cells is associated with disease stabilization in patients with p53 expressing advanced breast cancer; monitoring of serum YKL-40 and IL-6 as response biomarkers. Cancer Immunol Immunother. 2007; 56(9): 1485-1499, doi: 10.1007/s00262-007-0293-4, indexed in Pubmed: 17285289

11. Blank O, von Tresckow B, Monsef I, et al. Chemotherapy alone versus chemotherapy plus radiotherapy for adults with early stage Hodgkin lymphoma. Cochrane Database Syst Rev. 2017; 4: CD007110, doi: 10.1002/14651858.CD007110.pub3, indexed in Pubmed: 28447341.

12. Sher DJ, Mauch PM, Van Den Abbeele A, et al. Prognostic significance of mid- and post-ABVD PET imaging in Hodgkin's lymphoma: the importance of involved-field radiotherapy. Ann Oncol. 2009; 20(11): 18481853, doi: 10.1093/annonc/mdp071, indexed in Pubmed: 19541793.

13. Mikhaeel NG, Hutchings $M$, Fields PA, et al. Prognostic value of interim FDG-PET after two or three cycles of chemotherapy in Hodgkin lymphoma. Ann Oncol. 2005; 16(7): 1160-1168, doi: 10.1093/annonc/ mdi200, indexed in Pubmed: 15939713.

14. Barnes JA, LaCasce AS, Zukotynski K, et al. End-of-treatment but not interim PET scan predicts outcome in nonbulky limited-stage Hodgkin's lymphoma. Ann Oncol. 2011; 22(4): 910-915, doi: 10.1093/annonc/ mdq549, indexed in Pubmed: 20952598.

15. Kostakoglu L, Schöder H, Johnson JL, et al. Cancer Leukemia Group B. Interim [(18)F]fluorodeoxyglucose positron emission tomography imaging in stage I-II non-bulky Hodgkin lymphoma: would using combined positron emission tomography and computed tomography criteria better predict response than each test alone? Leuk Lymphoma. 2012; 53(11):2143-2150, doi: 10.3109/10428194.2012.676173, indexed in Pubmed: 22421007.

16. Zinzani PL, Rigacci L, Stefoni V, et al. Early interim 18F-FDG PET in Hodgkin's lymphoma: evaluation on 304 patients. Eur J Nucl Med Mol Imaging. 2012; 39(1): 4-12, doi: 10.1007/s00259-011-1916-8, indexed in Pubmed: 21894546

17. Terasawa T, Lau J, Bardet S, et al. Fluorine-18-fluorodeoxyglucose positron emission tomography for interim response assessment of advanced-stage Hodgkin's lymphoma and diffuse large B-cell lymphoma: a systematic review. J Clin Oncol. 2009; 27(11): 1906-1914, doi: 10.1200/ JCO.2008.16.0861, indexed in Pubmed: 19273713.

18. Gallamini A, Hutchings $M$, Avigdor A, et al. Early interim PET scan in Hodgkin lymphoma: where do we stand? Leuk Lymphoma. 2008; 49(4): 659-662, doi: 10.1080/10428190801888704, indexed in Pubmed: 18398732.

19. Akhtari M, Milgrom SA, Pinnix CC, et al. Reclassifying patients with early-stage Hodgkin lymphoma based on functional radiographic markers at presentation. Blood. 2018; 131(1): 84-94, doi: 10.1182/ blood-2017-04-773838, indexed in Pubmed: 29038339.

20. Bonadonna G, Bonfante V, Viviani $S$, et al. ABVD plus subtotal nodal versus involved-field radiotherapy in early-stage Hodgkin's disease: long-term results. J Clin Oncol. 2004; 22(14): 2835-2841, doi: 10.1200/ JCO.2004.12.170, indexed in Pubmed: 15199092.

21. Engert A, Schiller P, Josting A, et al. German Hodgkin's Lymphoma Study Group. Involved-field radiotherapy is equally effective and less toxic compared with extended-field radiotherapy after four cycles of chemotherapy in patients with early-stage unfavorable Hodgkin's lymphoma: results of the HD8 trial of the German Hodgkin's Lymphoma Study Group. J Clin Oncol. 2003; 21(19): 3601-3608, doi: 10.1200/ JCO.2003.03.023, indexed in Pubmed: 12913100.

22. Yahalom J, Illidge T, Specht L, et al. International Lymphoma Radiation Oncology Group, International Lymphoma Radiation Oncology Group, ILROG. Modern radiation therapy for Hodgkin lymphoma: field and dose guidelines from the international lymphoma radiation oncology group (ILROG). Int J Radiat Oncol Biol Phys. 2014; 89(4): 854-862, doi: 10.1016/j.jirobp.2013.05.005, indexed in Pubmed: 23790512.

23. Johnson DH, Schiller JH, Bunn PA. Recent clinical advances in lung cancer management. J Clin Oncol. 2014; 32(10): 973-982, doi: 10.1200/ JCO.2013.53.1228, indexed in Pubmed: 24567433. 